\title{
Introduction to the SoSyM theme issue on models and evolution
}

\author{
Dalila Tamzalit • Bernhard Schätz • Alfonso Pierantonio • \\ Dirk Deridder
}

Published online: 14 April 2013

(C) Springer-Verlag Berlin Heidelberg 2013

Software artifacts are subject to many sources of evolutionary pressure, which range from technical changes due to rapidly evolving technology platforms, to modifications caused by new requirements and insights emerging from the business domain. These modifications include changes at all levels, from requirements through architecture and design, to source code, documentation and test suites, and might affect any kinds of models. Therefore, adopting models, techniques, and tools for coping with and managing changes that accompany the evolution of software models is an essential discipline of Software Engineering.

\section{Models and evolution: great challenges}

With the increasing use of Model-Based Development in many domains (e.g., Automotive Software Engineering, Business Process Engineering, Software Architectures, Ser-

D. Tamzalit $(\varangle)$

LINA, University of Nantes, Nantes, France

e-mail: Dalila.Tamzalit@univ-nantes.fr

B. Schätz

Fortiss GmbH, Guerickestr. 25, 80805 München, Germany

e-mail: schaetz@fortiss.org

URL: http://www.fortiss.org/

\author{
A. Pierantonio \\ Department of Information Engineering, Computer Science \\ and Mathematics, Università degli Studi dell'Aquila, \\ 67100 L'Aquila, Italy \\ e-mail: alfonso.pierantonio@univaq.it \\ URL: http://www.di.univaq.it/alfonso/ \\ D. Deridder \\ Smals vzw, Fonsnylaan 20, 1060 Brussels, Belgium \\ e-mail: info@dirkderidder.be \\ URL: http://dirkderidder.be/
}

vice Oriented Engineering), models are becoming more and more primary artifacts of modern software engineering processes. By raising the level of abstraction and using concepts closer to the problem and application domain rather than the solution and technical domain, models become core assets and reusable intellectual property, being worthy of the effort in maintaining and evolving them. Therefore, increasingly models meet the same issues as traditional software artifacts, i.e., being subject to many kinds of changes, which range from rapidly evolving environments and platforms to the evolution of the functionality provided by the applications developed. These modifications again include changes at all levels, from requirements through architecture and design, to executable models, documentation and test suites. They typically affect various kinds of models including data models, behavioral models, domain models, source code models, goal models, etc., and also affect the management of models and metamodels. Therefore, coping with and managing the changes on models and metamodels is an essential aspect of Software Engineering as a discipline.

In addition, models can be adopted for a more abstract and expressive modeling of evolution in software systems. Indeed, they can successfully guide designers in dealing with software evolution by enforcing and reducing critical risks and important resources (e.g., costs, personnel, time) involved in software evolution, by raising abstraction. Models can thus help to direct software evolution.

\section{Models and evolution as a SoSyM theme issue}

This theme issue directly takes its roots from the international workshop dedicated to the topic "Models and Evolution" (http://www.models-and-evolution.com). The workshop has first been the fusion, in 2009, of two different workshops (MoDSE and MCCM) and has been proposed to the 
ACM/IEEE MoDELS conference. It is now in its fourth edition with MoDELS and experiences a growing success with around 50 attendees each year. This success shows the growing importance of issues around Models and Evolution, encouraging us to enforce it by proposing this dedicated theme issue.

After an open call for papers, we received around 30 submissions-making the preparation of this issue a great challenge. We have solicited close to 90 reviewers to ensure in-depth reviews and high-quality submissions. The theme issue would not have been possible without the excellent support of these reviewers, the help of the SoSyM editorial team, and the patience of the authors.

The objective of the theme issue is to provide a representative sample of advanced research being conducted in this area. Among the 30 submissions, 8 papers have been selected. They provide an overview of some of the most prominent and currently open issues, and identify some potential lines for further research... but there is still so much more to discover and propose! We are confident that the adventure will continue in the future.

Based on a long review process, we have selected the following eight papers for publication:

- In "Change Patterns: Co-evolving Requirements and Architecture" Koen Yskout, Riccardo Scandariato, and Wouter Joosen argue that patterns of co-evolution can be observed between intertwined pairs of artifacts, like the requirements specification and the architectural design. The paper introduces change patterns as a precise framework to systematically capture and handle change. The approach is based on model-driven engineering concepts and is accompanied by a tool-supported process.

- In "Evolution Styles: Foundations and Models for Software Architecture Evolution" Jeffrey Barnes, David Garlan, and Bradley Schmerl describe an approach for planning and reasoning about architecture evolution and by providing to architects a means to model prospective evolution paths and supporting analysis to select among these candidate paths. The paper also proposes an evolution path constraint specification language.

- In "Unique Identification of Elements in Evolving Software Models" Sven Wenzel presents an approach to identify and represent model elements and model fragments during their evolution by establishing a fine-grained history representation to describe their model evolution. An element can thus be identified in other revisions or variants of the model. Furthermore, changes over time can be captured.

- In "Extracting Models from Source Code in Software Modernization" Javier Cánovas Izquierdo and Jesús
García Molina address the problem of the extraction of models from general purpose programming languages (GPL) code of legacy systems. It is in fact an essential task in a model-based modernization process. For this objective, the paper proposes Gra2MoL, a DSL which is a text-to-model transformation language which can be applied to any code conforming to a grammar.

- In "Epsilon Flock: A Model Migration Language" Louis Rose, Dimitrios Kolovos, Richard Paige, Fiona Polack, and Simon Poulding propose a support for model migration: To update instance models in response to metamodel evolution. The paper proposes conservative copy, a style of model transformation and Epsilon Flock, a compact model-to-model transformation language tailored for model migration.

- In "Merging of EMF Models: Formal foundations" Bernhard Westfechtel presents a formal approach to both twoand three-way merging of models in the EMF framework. Context-free and context-sensitive rules are specified and a merge algorithm is developed that preserves consistency and produces consistent models.

- In "Adapting Transformations to Metamodel Changes via External Transformation Composition” Kelly Garces, Juan Manuel Vara, Frédéric Jouault, and Esperanza Marcos address impacts of the evolution metamodels on transformations. In fact, metamodels evolution impacts models as well as transformations. This paper focuses on the impact over transformations by adapting them by means of external transformation composition.

- In "A Semi-Formal Description of Migrating DomainSpecific Models with Evolving Domains" Tihamer Levendovszky, Daniel Balasubramanian, Anantha Narayanana, Chris van Buskirk, and Gabor Karsai address the migration of models, when their modeling language evolves, towards the evolved domain-specific modeling language (DSML). The paper proposes the Model Change Language (MCL) tool set that defines a domainspecific modeling language (DSML) to describe migration rules and to perform the migration automatically.

Acknowledgments This theme issue would not have been possible without the essential work done by the reviewers and the great patience of the authors. In particular, the authors deserve the most credit for making this theme issue possible by submitting their work, revising it according to the reviewers' comments, and patiently waiting for the final decisions. We are indebted to the reviewers for their timely efforts in evaluating all of the submitted papers, and for their help in choosing and improving the selected papers. Finally, our deepest thanks to the SoSyM editorial office and, in particular to Martin Schindler and Bernhard Rumpe. Martin Schindler spared no efforts to help us and to guide us during all this time. Thanks for the excellent support in preparing this theme section. 


\section{Author Biographies}

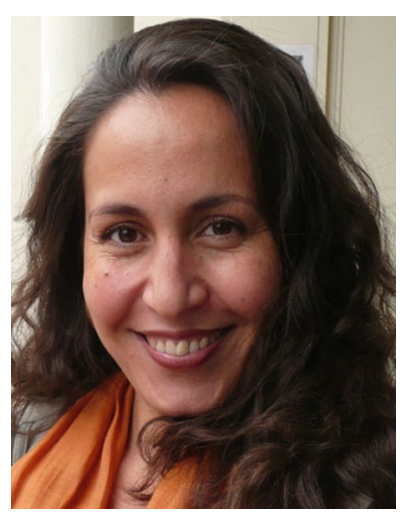

Dalila Tamzalit received her $\mathrm{Ph} . \mathrm{D}$. in computer science at the university of Nantes in 2000. She is an assistant professor at the University of Nantes in France since 2001. Her main research interest concerns software evolution foundations and methodologies. She published several peer-reviewed articles on this research topic in international journals and conferences. These last years, she focuses on Software Architecture Evolution. In addition to chairing the series of international workshops on Model-Driven Software Evolution (MoDSE) since 2007, Dalila Tamzalit is the head of the French CNRS research group on evolution, reuse and traceability of Information Systems (ERTSI) and co-founder and co-organizer of its dedicated workshop since 2002. She has also been co-organizer, program committee member and reviewer for French and international symposia, workshops, conferences and journals on Software Engineering and software evolution.

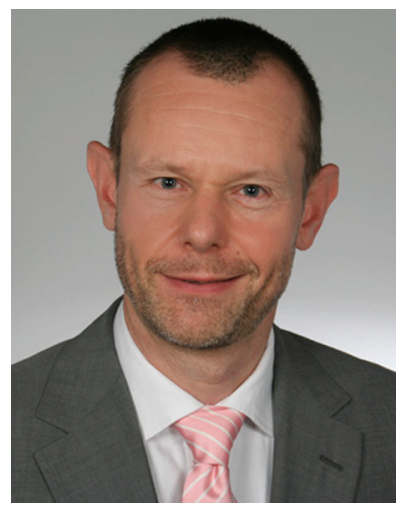

Bernhard Schätz received his Ph.D. in computer science at the TU München in 1998. He holds a lecturer position at TU München, Fakultät für Informatik, and acts as Deputy General Manager as well as heads the department for Software and System Engineering at the fortiss Research and Transfer Institute of the TU München. His work focuses at the development and application of methods for the engineering of reliable embedded software systems, especially by means of CASE tools for model-based development. He is the organizer of several regular events on model-based techniques. He was the organizer of the MODELS 2010 Doctoral Symposium and is the Program Co-Chair of MODELS 2013. Besides his academic activities, he works as a consultant.

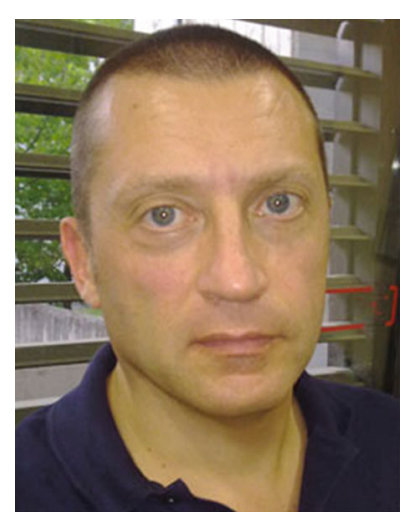

Alfonso Pierantonio is associate professor at the Department of Information Engineering, Computer Science and Mathematics of the University of L'Aquila (Italy) where he is currently director of the Master in Web Technology degree program. His current research interests include model-driven engineering, and in particular the management of co-evolution in metamodeling ecosystems, and bidirectionality in model transformations. He has been and is currently part of program and organization committees of conferences and is the chair of the steering committee of the International Conference on Model Transformation (ICMT). He co-edited several special issues on Model Transformations which appeared on relevant international journals.

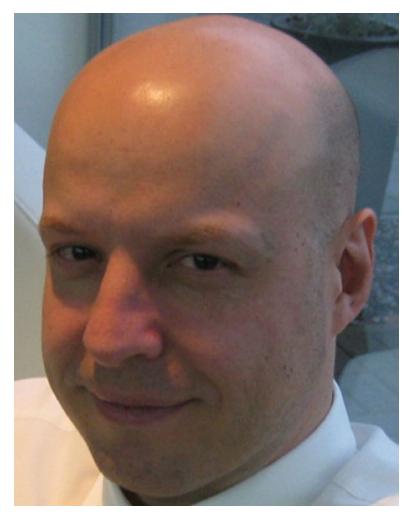

Dirk Deridder started his research career in 1997 at the Vrije Universiteit Brussel (Belgium) where he also received his $\mathrm{Ph} . \mathrm{D}$. in computer science. In 2011 he joined the research team of Smals vzw, where he is working on innovative software projects for the social sector and for the health sector of Belgium. His research covers modelbased software parameterization, model (co-)evolution, software variability engineering, and software architectures for extreme transaction processing. For 4 years he was one of the coordinators of the MoVES IAP-VI Network which grouped leading research teams active in the field of modeling, verification, and evolution of software. He has also been co-organizer and program committee member of several international symposia, workshops, and conferences. 